Received: 3 April 2017

Accepted: 10 August 2017

Published online: 29 August 2017

SCIENTIFIC REP

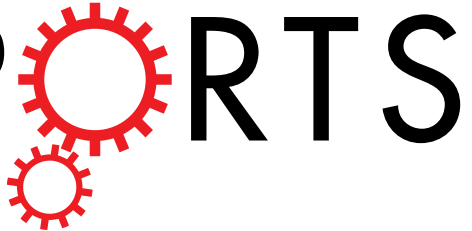

OPEN

\title{
Toward new gas-analytical multisensor chips based on titanium oxide nanotube array
}

Fedor Fedorov ${ }^{1}{ }^{1}$, Michail Vasilkov 2,3 , Andrey Lashkov ${ }^{3}{ }^{3}$, Alexey Varezhnikov ${ }^{3}$, Dirk Fuchs ${ }^{4}$, Christian Kübel $\mathbb{D}^{5}$, Michael Bruns ${ }^{6}$, Martin Sommer ${ }^{7}$ \& Victor Sysoev $\mathbb{D}^{3,8}$

Reliable environmental monitoring requires cost effective but highly sensitive and selective gas sensors. While the sensitivity of the sensors is improved by reducing the characteristic dimensions of the gas-sensing material, the selectivity is often approached by combining the sensors into multisensor arrays. The development of scalable methods to manufacture such arrays based on low-dimensional structures offers new perspectives for gas sensing applications. Here we examine an approach to produce multisensor array chips based on the $\mathrm{TiO}_{x}$ nanotube layers segmented by multiple $\mathrm{Pt}$ strip electrodes. We study the sensitivity and selectivity of the developed chip at operating temperatures up to $400^{\circ} \mathrm{C}$ towards organic vapors in the ppm range. The results indicate that the titania nanotubes are a promising material platform for novel cost-effective and powerful gas-analytical multisensor units.

The development of gas sensors with advanced characteristics is driven by the necessity of fast, reliable and cheap monitoring of the environment ${ }^{1}$ which is especially important for various emerging tasks ${ }^{2}$. The most remarkable success in that field is expected in material science of gas-sensitive elements primarily matured from technologies which allow one designing nano-dimensional structures ${ }^{3,4}$. The targeted sensor characteristics are higher sensitivity, stability and selectivity ${ }^{5,6}$ which should be combined with low production cost and low energy consumption of the final units ${ }^{6-8}$.

So far, metal oxide semiconductors are recognized as materials which fully meet the requirements of commercial gas sensors and have a great potential to advance further, mainly through application of nanotechnologies?. The general approach to greatly enhance their sensitivity is a reduction of the characteristic dimensions of the crystallites/grains composing the material ${ }^{10}$. This enhances the impact of surface processes on the total conductivity of the material as the ratio between Debye length and structure size increases ${ }^{11}$. However, 3D-nanosized oxide structures might be rather unstable especially at elevated temperatures which are conventionally employed to activate the sensors, particularly chemiresistors ${ }^{12}$, what questions their stability. In order to address this issue, quasi-1D structures such as nanowires, nanobelts or nanotubes (NTs) are considered to be the promising candidates for a new generation of gas sensors (see recent reviews ${ }^{13-15}$ and references therein). Still, the selectivity remains to be the most challenging aspect because metal oxide semiconductors are fundamentally unselective for chemisorbed gas species. Therefore, the most powerful way is to combine various sensors ${ }^{16}$, including those based on quasi $1 \mathrm{D}$ structures ${ }^{17}$, into arrays and process the obtained vector signal by pattern recognition techniques.

\footnotetext{
${ }^{1}$ Skolkovo Institute of Science and Technology, Skolkovo Innovation Center, 3 Nobel str., Moscow, Russian Federation. ${ }^{2} \mathrm{~V}$. A. Kotel'nikov Institute of RadioEngineering and Electronics of Russian Academy of Science, Saratov Branch, 38 Zelenaya str., Saratov, Russian Federation. ${ }^{3}$ Yuri Gagarin State Technical University of Saratov, 77 Polytechnicheskaya str., Saratov, Russian Federation. ${ }^{4}$ Institute for Solid-State Physics, Karlsruhe Institute of Technology (KIT), Hermann-von-Helmholtz-Platz 1, Eggenstein-Leopoldshafen, Germany. ${ }^{5}$ Institute of Nanotechnology and Karlsruhe Nano Micro Facility, Karlsruhe Institute of Technology (KIT), Hermann-vonHelmholtz-Platz 1, Eggenstein-Leopoldshafen, Germany. ${ }^{6}$ Institute for Applied Materials and Karlsruhe Nano Micro Facility, Karlsruhe Institute of Technology (KIT), Hermann-von-Helmholtz-Platz 1, Eggenstein-Leopoldshafen, Germany. ${ }^{7}$ Institute for Microstructure Technology, Karlsruhe Institute of Technology (KIT), Hermann-vonHelmholtz-Platz 1, Eggenstein-Leopoldshafen, Germany. ${ }^{8}$ Department of Functional Nanosystems and HighTemperature Materials, National University of Science and Technology "MISIS", Leninskiy pr. 4, Moscow, Russia. Correspondence and requests for materials should be addressed to F.F. (email: fedorov_fs@daad-alumni.de) orV.S. (email:vsysoev@sstu.ru)
} 


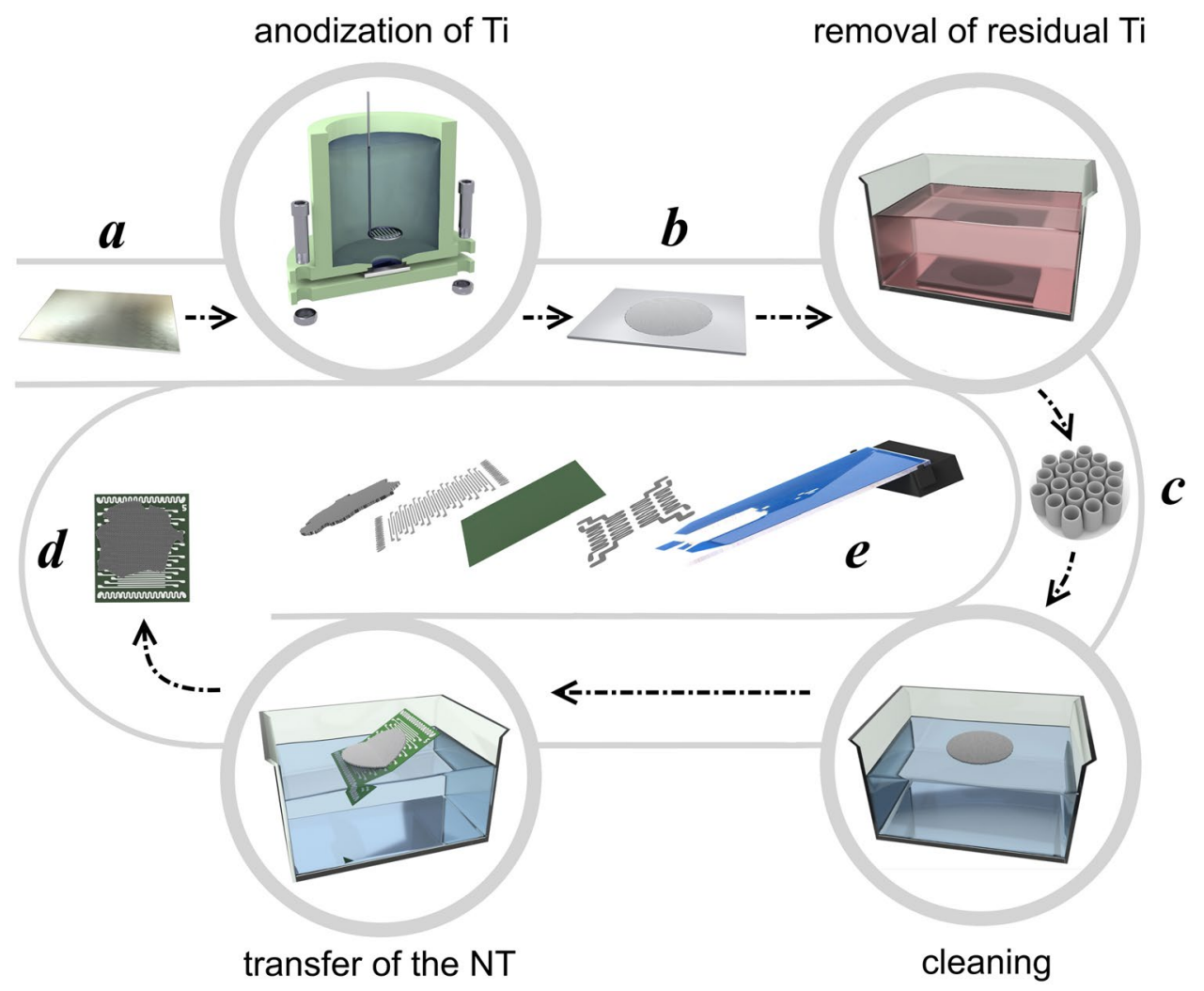

Figure 1. The scheme of fabrication the multisensor chip based on $\mathrm{TiO}_{\mathrm{x}} \mathrm{NT}$ array: (a-e) denote respectively $\mathrm{Ti}$ foil (a), Ti foil with as-prepared NT array (b), the extracted NT array (c), the NT array placed over the chip (d), the scheme of multisensor chip wired into 50-pin ceramic card (e).

The quasi 1D oxide structures can be synthesized via gas-based or solution-based techniques ${ }^{18-21}$. The second one is thought to be more suitable for mass production because of lower cost and better control the surface area to define the thickness at the nanoscale ${ }^{22}$. Among quasi-1-D structures, NTs are distinguished by their high specific surface due to their hollow structure with inner and outer surfaces that are exposed to the gases which makes it easier to fully deplete the oxide from free carriers due to surface localization under chemisorption and catalytic processes. That favors a "transduction function" in this material for sensor applications while a "receptor function" is still defined by a preferential oxide crystalline phase ${ }^{20,23}$.

In this report, we consider NTs of titanium dioxide which can be reliably fabricated using high yield proto$\operatorname{cols}^{24}$. The most cost effective and well established technology is anodization of titanium in $\mathrm{F}^{-}$containing electrolytes which is appropriate for mass-scaling ${ }^{20}$. By properly choosing the anodization bias one can tune fabricating oxide NT layers with precise control of the diameter, height, wall thickness of the tubes, inter-tube distance, thickness of the barrier layer, etc. ${ }^{25}$. This renders the material to be promising for chemiresistors using a conventional two-electrode design ${ }^{26-30}$. Although, a realization of these sensors is challenged by a negative influence of titanium underlayer which shunts NTs and leads to poor electrical contacts which reduces the reliability of the devices $^{31}$.

Despite some success in enhancing the sensitivity of the $\mathrm{TiO}_{2}$ NT-based sensors, the selectivity of gas identification remains unresolved. Even the approaches to functionalize the NTs by various dopants have not allowed one obtaining absolute sensitivity to only one gas ${ }^{32,33}$. Therefore, here we consider employing a multisensor approach $^{34}$ to be realized possibly in the cheapest design ${ }^{35}$ able in frames of microelectronic technologies by segmenting the pristine NT array by multiple co-planar electrodes. In particular, we demonstrate the capability of the chip to selectively detect few exemplary organic vapors at ppm concentrations.

\section{Results}

The process to employ the titanium dioxide NTs in the multisensor chip includes (Fig. 1) primarily an anodization of the titanium foil in accordance with known protocols $\mathrm{s}^{20,25,36,37}$. The as-anodized samples are further etched for removing the remained metal in absolute methanol/ $\mathrm{Br}_{2}$ solution ${ }^{38}$ in order to extract the pristine $\mathrm{TiO}_{\mathrm{x}} \mathrm{NT}$ array. The full details of the processes are given in Methods. Then, the $\mathrm{TiO}_{\mathrm{x}} \mathrm{NT}$ array is cleaned in pure alcohols and placed for floating over the distillated water surface. This array is then picked up and thoroughly positioned over the $\mathrm{Si} / \mathrm{SiO}_{2}$ multielectrode chip substrate ${ }^{39}$. The as-prepared chip is dried for few hours at room temperature in air.

The $\mathrm{Si} / \mathrm{SiO}_{2}$ chip substrate equipped with multiple Pt strip electrodes provides a number (up to 38) of NT array segments whose electrical/chemiresistive properties are measured independently. 

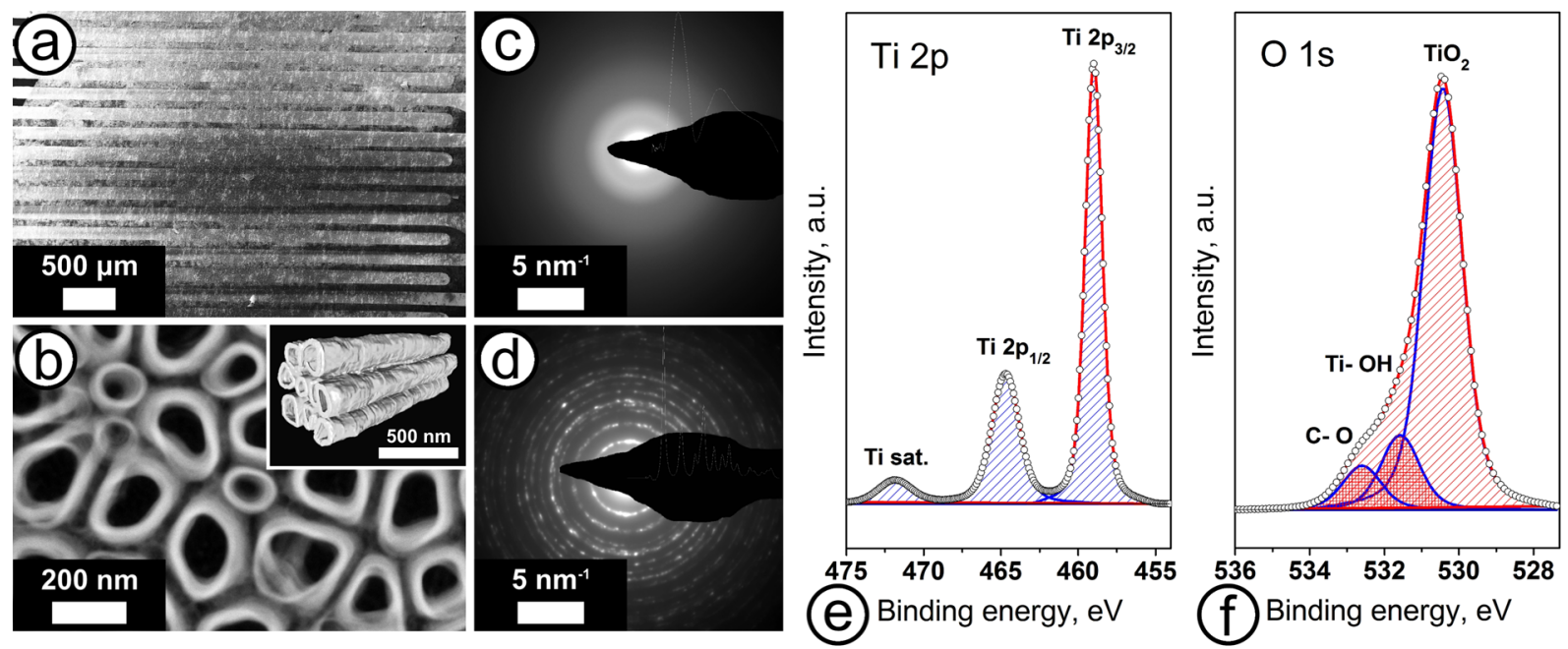

Figure 2. Characterization of the $\mathrm{TiO}_{\mathrm{x}} \mathrm{NT}$ arrays used in the multisensor chip: (a) SEM image taken of the chip surface covered with NT array; (b) HAADF-STEM image, the inset shows a surface rendering of the 3D STEM tomographic reconstruction viewed along the NT length; (c,d) SAED patterns of as-grown NTs and NTs annealed at $400^{\circ} \mathrm{C}$, respectively; (e,f) the XPS peaks related to Ti $2 \mathrm{p}(\mathbf{e})$ and $\mathrm{O}$ 1s (f) for the as-grown NTs, red lines show the envelope curves of all fitted peaks to compare with the experimental data (open circles).

Following the transfer into the chip the $\mathrm{TiO}_{\mathrm{x}} \mathrm{NT}$ array possesses a structural integrity as evidenced by scanning electron microscopy (SEM) inspection (Fig. 2a); the NTs are characterized with a distinct morphology (Fig. 2b).

The NTs are stand vertically to be almost perpendicular to the chip surface that means the NT open endings are positioned towards the outer atmosphere, including gas vapors under exposure. The mean NT inner diameter $D$ and the mean wall thickness $d$ are $102.7 \pm 18.1 \mathrm{~nm}$ and $14.5 \pm 5.2 \mathrm{~nm}$, respectively, while the NT density is ca. $4.3 \cdot 10^{9} \mathrm{~cm}^{-2}$. The SEM imaging of the array layer cross-section has proved the height of the NTs to be in range of $0.9-1.2 \mu \mathrm{m}$. This height ensures an easy gas access to NTs surface and according to Albu et al.$^{40}$ allows one staying adhered to chip surface and free of cracks under heating to temperatures higher than $300^{\circ} \mathrm{C}$ which are applied for the activation of chemiresistive effect in the NTs and chip operation.

In order to clarify the structure and morphology of NTs we have extracted the NT array out of the same batch as the ones employed in the chip for additional characterization by high-angle annular dark field-scanning transmission electron microscopy (HAADF-STEM) and X-ray photoelectron spectroscopy (XPS) techniques; the details are given in Methods.

Moreover, we have analyzed NTs in as-grown state and one following annealing at $400^{\circ} \mathrm{C}$ for $24 \mathrm{~h}$ in air atmosphere in order to simulate conditions of chip operation. The recorded HAADF-STEM images of both kinds of samples, as-grown and annealed ones, show that the $\mathrm{TiO}_{\mathrm{x}} \mathrm{NTs}$ are closely packed into an array where adjacent NTs have a distance of a few nm, which favors good gas access (Fig. $2 \mathrm{~b}$ and Supplementary). The annealing at $400^{\circ} \mathrm{C}$ does not change the tubular structure of the arrays. The 3D STEM tomographic reconstruction of the whole array indicates the existence of interconnections between NTs along their whole height (see inset at Fig. 2b and Supplementary videos). Some of the NTs bifurcate that increases their connection pathways and specific surface area. Selected area electron diffraction (SAED) supports that the $\mathrm{TiO}_{\mathrm{x}} \mathrm{NTs}$ are mostly amorphous in the as-grown state (Fig. 2c) to be organized into polycrystalline structure at anatase $\mathrm{TiO}_{2}$ phase after the annealing (Fig. 2d). The anatase crystal structure of annealed NTs is manifested by lattice strong reflections of (101), (103), (105) planes corresponding to d-values of $3.52 \AA$, $2.37 \AA, 1.75 \AA$ and reflections from other crystal planes (see Supplementary).

The XPS study of the as-grown NT array placed over $\mathrm{Au}$-coated $\mathrm{Si} / \mathrm{SiO}_{2}$ substrate yields that $\mathrm{Ti} 2 \mathrm{p}_{3 / 2}$ peak is positioned at $459.0 \mathrm{eV}$ and the corresponding $\mathrm{O} 1 \mathrm{~s}$ peak is observed at $530.3 \mathrm{eV}$, both in a good agreement with literature references ${ }^{41}$. Surface $\mathrm{Ti}-\mathrm{OH}$ groups are indicated by the $\mathrm{O} 1 \mathrm{~s}$ peak at $531.2 \mathrm{eV}$ which cannot be seen in the energy region related to $\mathrm{Ti} 2 \mathrm{p}$ due to a low intensity. The weak higher binding energy $\mathrm{O} 1 \mathrm{~s}$ peak at $532.4 \mathrm{eV}$ corresponds to C-O groups and corroborates with C 1s signal from surface contaminations component observed at $289.1 \mathrm{eV}$ (Fig. 2e, Supplementary). The XPS survey spectrum also suggests the presence of fluorine traces in the as-grown NTs which stem from electrolyte residues and are removed after annealing of NTs at $400{ }^{\circ} \mathrm{C}$ in accordance with literature ${ }^{42}$. Still, other studies support possible fluctuating of the foreign elements, $[\mathrm{F}]$ and $[\mathrm{C}]$, at low concentrations in $\mathrm{NTs}^{43}$ and the existence of Ti in different oxidation state close to $\mathrm{Ti} / \mathrm{TiO}_{2}$ interface $^{44}$. Such minor variations of structure and elemental composition in NTs have been out of XPS detection in our samples but still could contribute to the observed differences in local electrical and gas-sensitive properties along NT array membrane as further discussed.

Based on the relative intensity of the $\mathrm{Ti} 2 \mathrm{p}_{3 / 2}$ and $\mathrm{O} 1 \mathrm{~s}$ peaks, the surface $[\mathrm{O}] /[\mathrm{Ti}]$ atomic ratio is calculated to be about 1.92 . The annealing of NTs at $400{ }^{\circ} \mathrm{C}$ in air increases the oxygen content towards attainment 

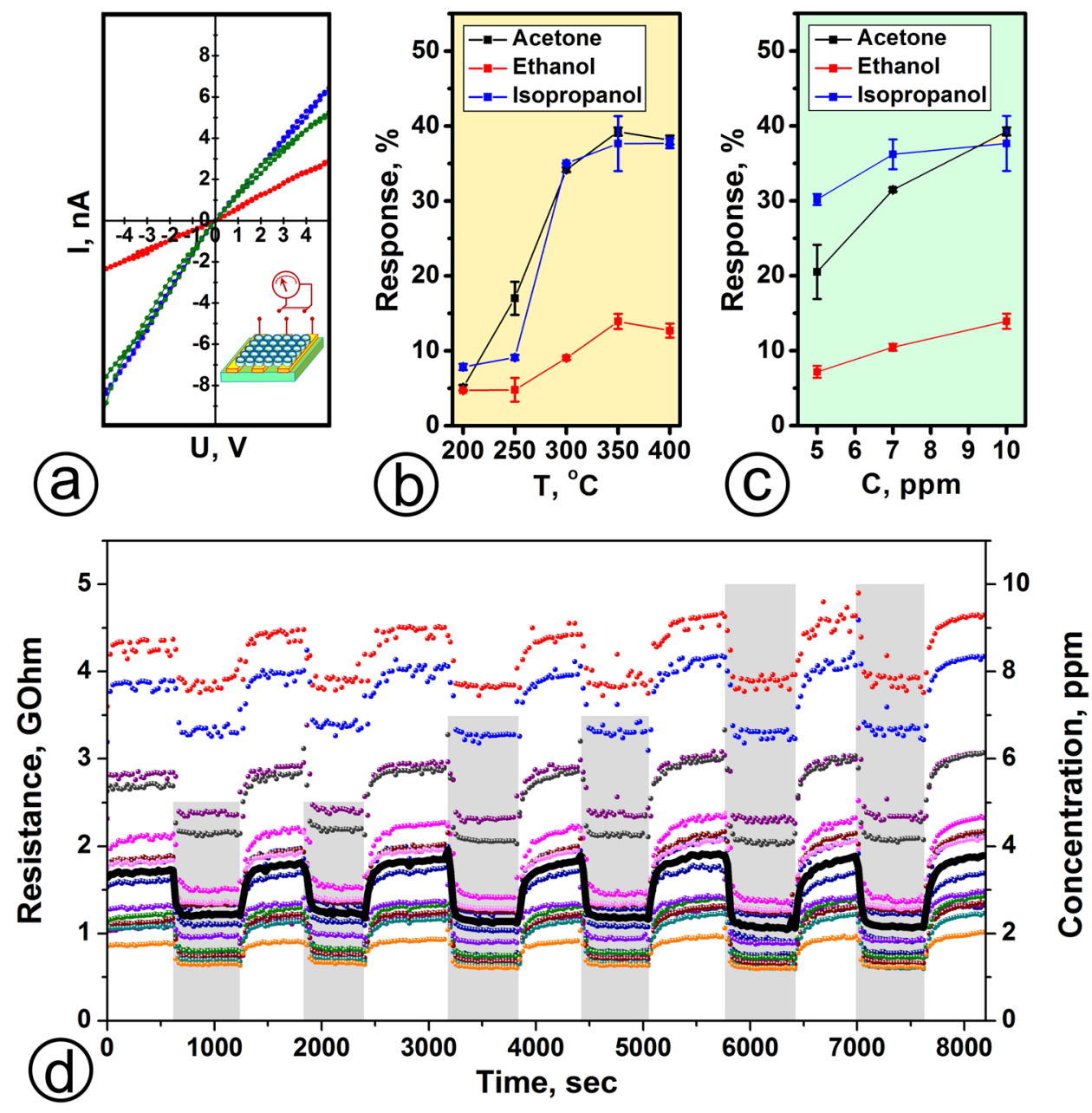

Figure 3. The electrical characterization of $\mathrm{TiO}_{\mathrm{x}} \mathrm{NT}$ array based multisensor chip: (a) $\mathrm{I}-\mathrm{V}$ curves recorded from three exemplary segments under constant flow $(100 \mathrm{sccm})$ of laboratory air at $350^{\circ} \mathrm{C}$; (b) the dependence of chip segments response to $10 \mathrm{ppm}$ of ethanol, isopropanol, and acetone on operating temperature; (c) the dependence of chip segments response on vapor (ethanol, isopropanol, and acetone) concentration in air at $350^{\circ} \mathrm{C}$; (d) the variations of the chip segments resistances, $R(t)$, to pulses of isopropanol at different ppm concentrations under operating temperature of $350^{\circ} \mathrm{C}$; thick black line denotes the segments median resistance.

of the stoichiometry ratio equal to approx. 1.95. Obviously, even in annealed state the $\mathrm{TiO}_{\mathrm{x}} \mathrm{NTs}$ appear to be non-stoichiometric with an oxygen deficiency which is a major prerequisite to observe a chemiresistive effect ${ }^{45,46}$.

In order to activate and stabilize the as-prepared $\mathrm{NTs}^{47}$ for gas sensing tests, we have primarily heated the NT-based multisensor chip up to $400^{\circ} \mathrm{C}$ for $24 \mathrm{~h}$ in air atmosphere and then carried out the isothermal electrical measurements in RT $-400^{\circ} \mathrm{C}$ range. At operating temperatures lower than $200^{\circ} \mathrm{C}$ the NT array segment resistance over the chip was too high to be read out by our electronic measuring units (see Methods). In the temperature range of $200-400^{\circ} \mathrm{C}$, the I-V curves measured for the NT array segments have been found to be nearly linear (Fig. 3a) in air and in the test vapors (isopropanol, ethanol and acetone) mixed with air. This reasonably indicates a good contact between NTs and the electrodes without significant Schottky barriers. A temperature increase from $200^{\circ} \mathrm{C}$ to $400^{\circ} \mathrm{C}$ leads to a drop of the mean resistance of the NT array segments in air from tens of GOhms to hundreds MOhms (see Supplementary) following a semiconducting behavior. The activation energy calculated from the conductance-vs-temperature curve is around $0.44 \mathrm{eV}$ which correlates well with the reported values for polycrystalline $\mathrm{TiO}_{2}{ }^{48}$. This value originates from the potential barriers between adjacent $\mathrm{NTs}^{49}$ and/or the energy levels of local states related to oxygen vacancies ${ }^{45,46}$ in the oxide gap which are involved in electron exchange processes at the NT surface.

Following the chip exposure towards isopropanol, ethanol and acetone vapors, 5-10 ppm concentrations, in mixture with laboratory air at 30-35 rel. \% humidity, we have observed a clear chemiresistive effect in NTs at all operating temperatures in $200-400^{\circ} \mathrm{C}$ range. The maximum gas response as a relative resistance change, $\Delta R /$ $R_{\text {air }}$, in the vapor-enriched air has been observed at $350^{\circ} \mathrm{C}$ (Fig. $3 \mathrm{~b}$ ). At this temperature, the median NT array segments response to isopropanol and acetone of $10 \mathrm{ppm}$ concentration is close to $38 \%$ while one to $10 \mathrm{ppm}$ of ethanol is about $14 \%$. The concentration dependence of the gas response at this temperature follows Freundlich isotherm, $\Delta R / R_{\text {air }}=k C^{n}$, where power low index $n$ for isopropanol, ethanol and acetone is about 0.4, 0.9, 0.6, 


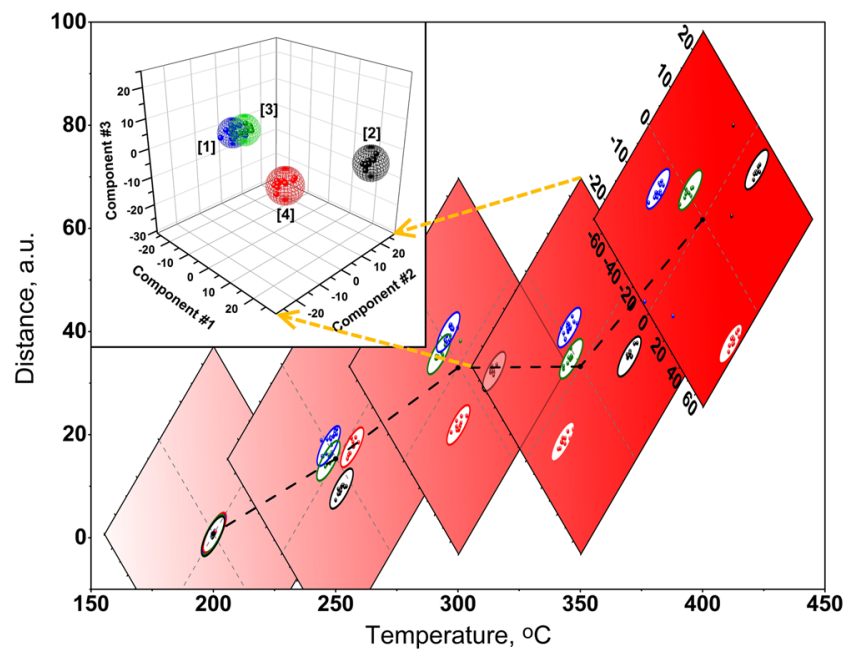

Figure 4. The LDA processing of vector responses of NT array-based multisensor chip to test organic vapors, $10 \mathrm{ppm}$ concentration: dependence of the average Mahalanobis distance between the centers of vapor-related LDA clusters as a function of operating temperature. The projections of the generated LDA space into primary two-component plane are centered at the obtained points referring to Mahalanobis distance. The inset is the LDA 3-component diagram built for the chip operated at $350^{\circ} \mathrm{C}$ : 1-air,2-acetone,3-ethanol,4-isopropanol. All the spheres are calculated at 0.95 confidence level to frame data training clusters; the points are related to the test responses.

correspondingly (Fig. 3c) which is quite typical for the most metal oxides ${ }^{50}$. The observed signals have been stable, at least for few days (see Supplementary).

These remarkable differences in responses to these gases are caused by variations in chemical adsorption and dissociation of these molecules at the NT surface that provides a background to selective recognition of these vapors by multisensor chip array as further discussed. Here, it is worth noting that the change of the median resistance exceeds $8 \%$ to $5 \mathrm{ppm}$ of isopropanol already at $200^{\circ} \mathrm{C}$ what indicates rather low gas detection limit for the developed multisensor. Figure $3 \mathrm{~d}$ shows the typical reduction of resistance of exemplary segments of $\mathrm{TiO}_{\mathrm{x}} \mathrm{NT}$ array when exposed to isopropanol vapors at $350^{\circ} \mathrm{C}$ which is optimal one to observe the chemiresistive effect. The similar data have been observed in case of other test vapors at different temperatures.

It is clear that the organic vapor traces substantially decrease the resistance of the segments in accordance with $n$-type semiconductor response of non-stoichiometric $\mathrm{TiO}_{2}$. Still, the spatial inhomogeneous local morphology of the NT array across the chip segments results in the variation of both the resistance and gas sensitivity. It allows one employing it to build a vector signal whose components are constitued by signals from the gas-sensitive segments in the chip and approach the gas selectivity in the framework of known multisensor concept ${ }^{16}$. In order to process the NT-based multisensor vector response to the test vapors we have applied the Linear Discriminant Analysis (LDA) which maximizes the vector variations induced by different vapors and minimizes the vector scatters under exposure to certain vapor ${ }^{51}$. The confidence probability to build the LDA clusters related to gases has been installed to be equal to 0.95 . By these means, we have tested all the multisensor chip vector signals recorded at operating temperatures in $200-400^{\circ} \mathrm{C}$ range (Fig. 4). In order to build the training set in the LDA space the sampling of 10 vector signals recorded under each atmosphere has been employed while other recorded signals were served for testing. Here, we define the selectivity as an average Mahalanobis distance between clusters in the LDA space ${ }^{52}$. This parameter allows us to estimate quantitatively the quality of gas recognition under various working conditions of the chip. As we found, the selectivity strongly depends on the operating temperature (Fig. 4b,c). The temperature enhancement from $200^{\circ} \mathrm{C}$ to $400^{\circ} \mathrm{C}$ results in 2 -order change in the mean Mahalanobis distance between the clusters from ca. 0.8 to 61.7, correspondingly, in the 3-dimensional LDA coordinate system. This selectivity estimation clearly correlates with gas response behavior with temperature up to $350^{\circ} \mathrm{C}$ : the higher response means higher selectivity. However, $400^{\circ} \mathrm{C}$ is even more favorable for selectivity while gas response slightly reduces. These data indicate that the operating temperature facilitates not only the at-surface charge-exchange processes but also the minor variations of potential barriers observed in NT-to-NT percolation paths in the array.

While the electron exchange between the chemisorbed species and semiconducting oxide surface depends mostly on the material itself, the micro- and mesostructure of the layer, its porosity and characteristic geometry substantially contribute to the overall NT array resistance measured at different environments ${ }^{53}$. Basically, the two dominant fundamental mechanisms, the depletion of the oxide material by free electrons and existence of potential barriers in contacts between the NTs, interplay in the chemiresistive response of the NT array. Under air conditions the NT surface is fully covered by oxygen and hydroxyl chemisorbed species which localize the electrons from the oxide conduction band ${ }^{54}$. This induces at-surface space charge region whose width in the oxide is comparable with the average NT wall thickness and crystallites. Taking the obtained NT array resistance values measured in air conditions we assess ${ }^{55}$ the free electron concentration, $n=\frac{g}{e \mu h R_{s}}$, where $e$ is elementary charge, $h$ 


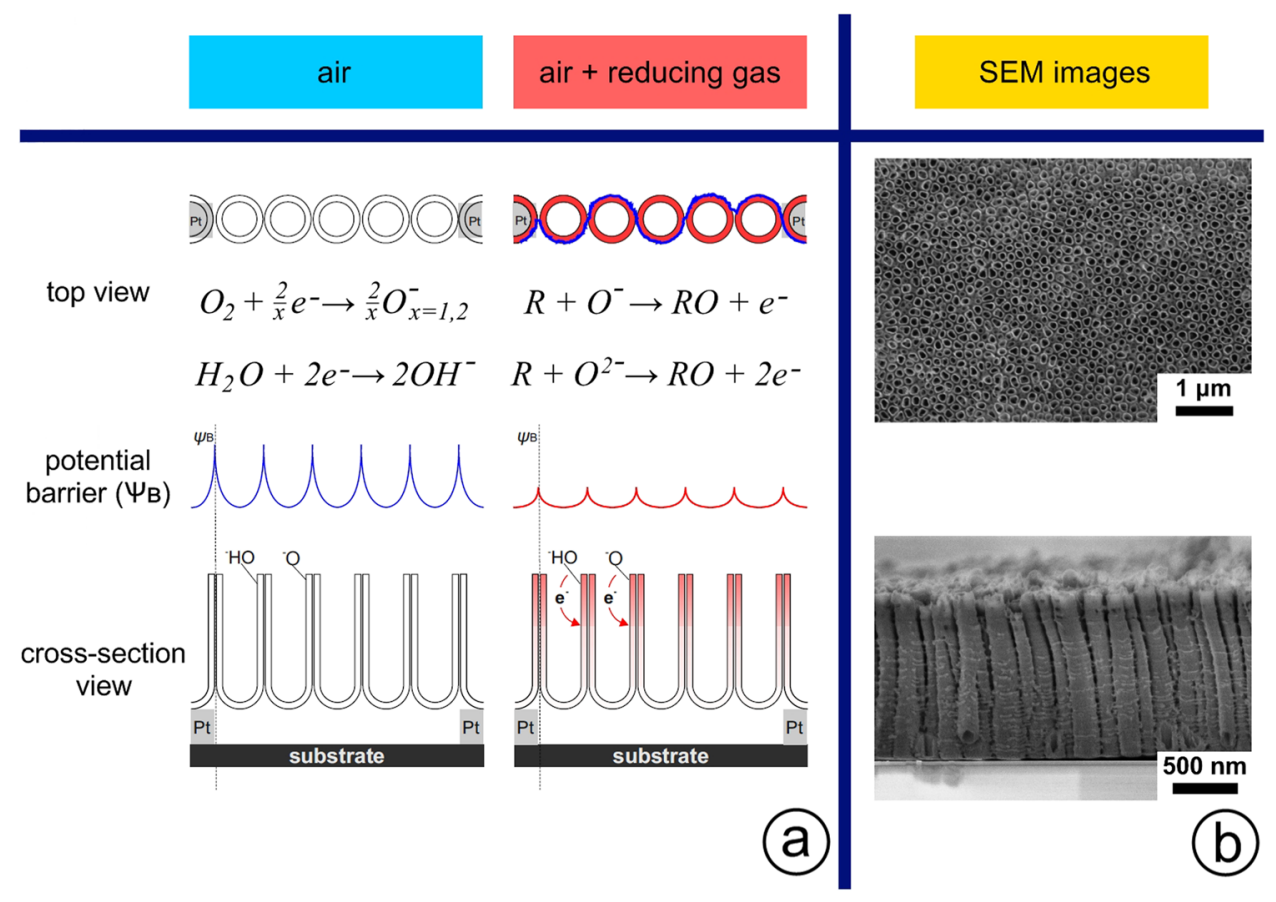

Figure 5. A schematic representation to show the change in the conductivity and conduction pathway for electrons through $\mathrm{TiO}_{\mathrm{x}} \mathrm{NT}$ array at two environments - air and mixture of air and reducing agents: (a) the modification of potential barrier heights following the adsorption of the species of different origin and their surface reactions; (b) SEM images of the NT array from top and side views corresponding to the presented scheme.

the tube height, $R_{s}$ the NT array sheet surface resistance per square (see Supplementary), $\mu$ the electron mobility, which we consider equal to approx. $0.1 \mathrm{~cm}^{2} / \mathrm{Vs}$, characteristic for polycrystalline $\mathrm{TiO}_{2}{ }^{56}$. The geometry coefficient $g=\frac{(D+2 d)^{2}}{2 d(D+d)}$ accounts for reduction of in-fact volume of the NT array sheet when compared to compact layer due to the porous tubular geometry and confining the electron pathway within the NT walls. Considering the obtained magnitudes of $D$ and $d$ the estimated value of $g$ is equal to approx. 5.1. For instance, at operating temperature of $350^{\circ} \mathrm{C}$ one gets $n$ equal to approx. $3.3 \pm 0.4 \cdot 10^{13} \mathrm{~cm}^{-3}$ that is lower by $3-4$ orders of magnitude when compared to concentrations reported for a single anatase NT measured in vacuum conditions ${ }^{57}$. The corresponding Debye length, $L_{d}=\sqrt{\frac{\varepsilon \varepsilon_{0} k T}{\rho^{2} \eta}}$, where $k$ is Boltzmann constant, $\varepsilon_{0}$ the electric constant, $\varepsilon$ the dielectric permittivity of anatase (equal to approx. 31) s8, $59^{e}, T$ the operating temperature, at these conditions goes to micrometer range that obviously exceeds the NT wall. Therefore, under air conditions the Fermi level in the NTs is pinned at the local states induced by oxidizing/hydroxyl adspecies in the oxide gap that is ordinarily described as a flat-band approximation $^{60}$. It results in remarkable reduction both the free electron concentration in the NT wall and the electron mobility due to enhancing the potential barrier, $\psi_{B}$, between $\mathrm{NTs}^{61}$. Consequently, this originates a high NT array resistance observed. So, the electron transport in such structures is mainly governed by carrier-depleted NT walls contacting each other like in the case of grains in polycrystalline sample ${ }^{54}$.

The exposure to reducing organic vapors releases the at-surface localized electrons to conductance band and decreases $\psi_{B}^{50,55}$. We consider the only electronic component of the oxide conductance because the operating temperatures below $400{ }^{\circ} \mathrm{C}$ are still not enough to stimulate a significant exchange between surface and bulk of NTs by intrinsic defects ${ }^{58}$. So, under application of electric field the electrons go through NT adjacent walls whose potential barrier height and "tunneling density" depend on a gas environment (Fig. 5). Thus, the whole NT array looks like a network of percolating tubes where analyte molecules modify the conduction pathways upon interaction with NT surfaces and junctions. The surface reactions between the gas molecules (see Fig. 5a) and/or their direct catalysis include a dissociation accompanied by an exchange of charged species that involves different amounts of charge localized by chemisorbed species.

This explains the higher response of the NTs towards isopropanol and acetone vapors when compared to one versus ethanol. Moreover, a stochastic combination of NT-to-NT contacts at each chip segment underlies the diversity of their gas-sensing properties along the array that allows us to build the chip vector signal selective to gases.

In conclusion, the obtained data clearly show that $\mathrm{TiO}_{\mathrm{x}} \mathrm{NT}$ array is a promising material to employ not only for the development of conventional gas sensors but gas-analytical multisensor chips as well. We have introduced the approaches to build up such chips which include primarily an anodization of titanium into oxide nanotubular layer with following removal of residual metal and secondly the transfer of the layer over the multielectrode $\mathrm{Si} / \mathrm{SiO}_{2}$ chip substrate by a method similar to Langmuir-Blodgett one. The tested samples of NT array-based multisensor chip have exhibited high sensitivity towards organic vapors in ppm range. The intrinsic stochastic 
inhomogeneities of the array along the chip yield obtaining the selectivity of gas discrimination by processing the multisensor vector signal generated by chip segments via pattern recognition technique. Still, the chip design allows one further advancing the selectivity by application of inhomogeneous heating and/or other external modifications.

\section{Methods}

$\mathrm{TiO}_{\mathbf{x}}$ NT array fabrication. $\mathrm{TiO}_{\mathrm{x}} \mathrm{NTs}$ are prepared by anodization of $\mathrm{Ti}, 99.6 \%$ (Sigma-Aldrich) foil of $127 \mu \mathrm{m}$ thickness. Prior to anodization the titanium foil has been immersed into acetone and ethanol, both for $10 \mathrm{~min}$, in sequence under sonification and then rinsed in deionized water $(18.2 \mathrm{M} \Omega \mathrm{cm})$. The titanium foil is characterized with a roughness less than $0.7 \mu \mathrm{m}$ measured with surface profiler (Veeco DekTak 150). The electrochemical anodization has been performed in a Teflon ${ }^{\circledR}$ cell in two-electrode configuration with a platinum mesh as cathode biased at $30 \mathrm{~V}$ for 1 hour at room temperature using electrolyte based on glycerol/water 3:1 solution with addition of $0.8 \% \mathrm{NH}_{4} \mathrm{~F}$ (Supplementary Figure 1). All chemicals have been provided to be at least of analytical purity (Sigma-Aldrich). The electrode area is separated by O-ring rubber to expose a Ti surface, $0.79 \mathrm{~cm}^{2}$, to the electrolyte. The anodization experiments have been carried out using a high-voltage potentiostat (Keithley 2410 source meter) connected to a digital multimeter (Keithley 2700 multimeter /data acquisition system) interfaced to personal computer (PC). The $\mathrm{TiO}_{\mathrm{x}} \mathrm{NT}$ array is afterwards rinsed in ethanol and is dried at room temperature for $0.5-1 \mathrm{~h}$. The Ti sublayer remains have been selectively dissolved in an absolute methanol/ $\mathrm{Br}_{2}$ solution, $1: 9$ vol., at $20^{\circ} \mathrm{C}$ for 2 hours according to ref. 38 . Then, the obtained layer has been positioned over the $\mathrm{Si} / \mathrm{SiO}_{2}$ chip equipped with multiple planar electrodes. Thermal treatment of titania NTs has been performed in PEO-601 oven (ATV Technologie $\mathrm{GmbH}$ ) in air atmosphere at $400^{\circ} \mathrm{C}$ during $24 \mathrm{~h}$. Following the annealing the samples have been rinsed by distilled water and dried in air.

$\mathrm{TiO}_{\mathbf{x}}$ NT array characterization. The morphological characterization of the NT array has been carried out using a scanning electron microscope (AURIGA ${ }^{\circledR}$ CrossBeam ${ }^{\circledR}$ workstation, Carl Zeiss) equipped with an energy dispersive X-ray (EDX) detector. The NT structure has been studied by transmission electron microscopy (TEM) using an aberration (image) corrected Titan 80-300 (FEI Company) equipped with a US1000 (Gatan) CCD camera. After mechanical removing the $\mathrm{TiO}_{\mathrm{x}} \mathrm{NTs}$ from the substrate, they have been dispersed in $n$-heptane by sonification and deposited on a carbon coated Cu grid (Quantifoil) for the TEM analysis. For an electron tomographic characterization in HAADF-STEM mode, $15 \mathrm{~nm}$ gold nanoparticles (University of Utrecht) were added for alignment and a tilt-series recorded from $-60^{\circ}$ to $60^{\circ}$ in $2^{\circ}$ steps. The tilt-series alignment was performed using IMOD (University of Colorado) and the 3D volume reconstructed using the SIRT algorithm implemented in Inspect3D (FEI Company). The Supplementary video shows a volume rendering of the 3D structure of the NT array morphology and a surface rendering of a selected part of the 3D reconstruction prepared in Amira (FEI Company).

The X-ray photoelectron spectroscopy (XPS) measurements have been conducted using a K-Alpha+ XPS spectrometer (Thermo Fisher Scientific, East Grinstead, UK). Data acquisition and processing using the @ Thermo Avantage software are described elsewhere ${ }^{62}$. We have utilized Si substrate with sputtered Au layer, approx. $1 \mu \mathrm{m}$ thick, to place the titania NT array after the metallic Ti is removed. All samples have been measured using a microfocused monochromated $\mathrm{Al} \mathrm{K \alpha} \mathrm{X}$-ray source of 30-400 $\mu \mathrm{m}$ spot size. The K-Alpha charge compensation system is employed during analysis using electrons of $8 \mathrm{eV}$ energy and low-energy argon ions to prevent any localized charge build-up. The spectra are fitted with one or more Voigt profiles; the binding energy uncertainty is $\pm 0.2 \mathrm{eV}$. The analyzer transmission function, Scofield sensitivity factors ${ }^{63}$ and effective attenuation lengths (EALs) for photoelectrons are applied for quantification. EALs are calculated using the standard TPP-2M formalism ${ }^{64}$. All spectra have been referenced to the $\mathrm{C} 1 \mathrm{~s}$ peak of hydrocarbon at $285.0 \mathrm{eV}$ binding energy controlled by means of the well-known photoelectron peaks of metallic $\mathrm{Cu}, \mathrm{Ag}$, and $\mathrm{Au}$.

The multielectrode substrate as a platform for the multisensor chip. We have utilized multielectrode chip based on $\mathrm{Si} / \mathrm{SiO}_{2}$ substrate equipped with $39 \mathrm{Pt}$ strip electrodes which define, if necessary, 38 segments of the NT array as a maximum number ${ }^{39}$. The inter-electrode distance is about $80 \mu \mathrm{m}$, electrode width is $100 \mu \mathrm{m}$, and the electrodes are $1 \mu \mathrm{m}$ thick. All the electrodes have a length of about $4 \mathrm{~mm}$ that defines the surface area of about $4 \times 8 \mathrm{~mm}^{2}$ whose part or total is covered by NT array. The frontside of the substrate has two meander-shaped Pt thermoresistiors at the edges to monitor the operating temperature which is adjusted by four meander heaters located at the backside. The operating temperature is maintained by corresponding PC-driven electronics unit with accuracy of $1^{\circ} \mathrm{C}$ while the temperature distribution at isothermal chip operation can be of $\pm 10^{\circ} \mathrm{C}^{52}$.

We have tested a number of chips in preliminary studies, the reported data belong to one exemplary chip.

Gas response measurements with NT array multisensor chip. The response of multisensor chip equipped with $\mathrm{TiO}_{\mathrm{x}} \mathrm{NT}$ array towards different organic vapors has been studied using a setup which includes home-made data acquisition unit, test gas generator, gas delivery tubes and electronic unit to maintain and monitor the chip operating temperature. Data acquisition module (National Instruments USB-6259) together with current pre-amplifier (SRS, SR570) has provided the resistivity measurements up to tens GOhms of each segment in the chip. To read out the total array resistances we have employed a multiplexing card for switching between the segments. Test vapor mixtures with laboratory air have been obtained with gas generator (Owlstone, UK) which employs heated permeation tubes containing a test liquid to yield vapors of several ppm of concentration under equilibrium conditions. The vapors of the same reducing origin, alcohols and acetone, have been utilized as test ones. The vapors have fed the chip by series of $10 \mathrm{~min}$ duration interrupted by pulses of pure laboratory air for 10-25 minutes to ensure the recovery of the chip segment resistances. The concentration of vapors in mixture with air has been maintained to be $5,7,10 \mathrm{ppm}$ for each one. The laboratory air is delivered by an oil-free 
compressor as is to be of normal humidity level in range of approx. 30-35 rel. \%. The all gases have been supplied in a flow mode with $100 \mathrm{sccm}$ rate. We have utilized only the data for NT array segments with resistivity less than $10 \mathrm{GOhm}$ at maximum operating temperature of $400^{\circ} \mathrm{C}$. Therefore, 16 segments of the multielectrode chip have been considered in the reported data.

\section{References}

1. Lewis, A. \& Edwards, P. Validate personal air-pollution sensors. Nature 535, 29-31 (2016).

2. Lichtenstein, A. et al. Supersensitive fingerprinting of explosives by chemically modified nanosensors arrays. Nat. Commun. 5, 4195 (2014).

3. Zhang, A. \& Lieber, C. M. Nano-bioelectronics. Chem. Rev. 116, 215-257 (2016).

4. Thamri, A. et al. MHDA-functionalized multiwall carbon nanotubes for detecting non-aromatic VOCs. Sci. Rep. 6, 35130 (2016).

5. Yamazoe, N. Toward innovations of gas sensor technology. Sensors Actuators B Chem. 108, 2-14 (2005).

6. Di Natale, C., Paolesse, R., Martinelli, E. \& Capuano, R. Solid-state gas sensors for breath analysis: a review. Anal. Chim. Acta 824, 1-17 (2014).

7. Strelcov, E. et al. Evidence of the self-heating effect on surface reactivity and gas sensing of metal oxide nanowire chemiresistors. Nanotechnology 19, 355502 (2008).

8. Semancik, S. et al. Microhotplate platforms for chemical sensor research. Sensors Actuators, B Chem. 77 (2001).

9. Korotchenkov, G. \& Sysoev, V. V. In Chemical sensors: comprehensive sensor technologies, Volume 4: Solid state devices (ed. Korotchenkov, G.) 53-186 (Momentum Press, LLC, 2011).

10. Das, S. \& Jayaraman, V. $\mathrm{SnO}_{2}$ : a comprehensive review on structures and gas sensors. Prog. Mater. Sci. 66, 112-255 (2014)

11. Barsan, N., Koziej, D. \& Weimar, U. Metal oxide-based gas sensor research: How to? Sensors Actuators B Chem. 121, 18-35 (2007).

12. Sysoev, V. V. et al. Percolating $\mathrm{SnO}_{2}$ nanowire network as a stable gas sensor: direct comparison of long-term performance versus $\mathrm{SnO}_{2}$ nanoparticle films. Sensors Actuators B Chem. 139, 699-703 (2009).

13. Comini, E. et al. Quasi-one dimensional metal oxide semiconductors: Preparation, characterization and application as chemical sensors. Prog. Mater. Sci. 54, 1-67 (2009).

14. Arafat, M. M., Dinan, B., Akbar, S. A. \& Haseeb, A. S. M. A. Gas sensors based on one dimensional nanostructured metal-oxides: a review. Sensors (Basel). 12, 7207-58 (2012).

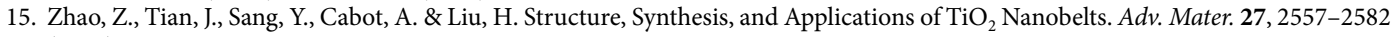
(2015).

16. Persaud, K. \& Dodd, G. Analysis of discrimination mechanisms in the mammalian olfactory system using a model nose. Nature 299, 352-355 (1982)

17. Sysoev, V., Button, B., Wepsiec, K., Dmitriev, S. \& Kolmakov, A. Toward the nanoscopic 'electronic nose': hydrogen vs carbon monoxide discrimination with an array of individual metal oxide nano- and mesowire sensors. Nano Lett. 6, 1584-1588 (2006).

18. Dasgupta, N. P. et al. Semiconductor nanowires - synthesis, characterization, and applications. Adv. Mater. 26, 2137-2184 (2014).

19. Devan, R. S., Patil, R. A., Lin, J.-H. \& Ma, Y.-R. One-dimensional metal-oxide nanostructures: recent developments in synthesis, characterization, and applications. Adv. Funct. Mater. 22, 3326-3370 (2012).

20. Roy, P., Berger, S. \& Schmuki, P. TiO nanotubes: synthesis and applications. Angew. Chemie Int. Ed. 50, 2904-2939 (2011).

21. Zwilling, V., Aucouturier, M. \& Darque-Ceretti, E. Anodic oxidation of titanium and TA6V alloy in chromic media. An electrochemical approach. Electrochim. Acta 45, 921-929 (1999).

22. Regonini, D., Bowen, C. R., Jaroenworaluck, A. \& Stevens, R. A review of growth mechanism, structure and crystallinity of anodized $\mathrm{TiO}_{2}$ nanotubes. Mater. Sci. Eng. R Reports 74, 377-406 (2013).

23. Guo, W. et al. Systematic investigation on the gas-sensing performance of $\mathrm{TiO}_{2}$ nanoplate sensors for enhanced detection on toxic gases. Mater. Res. Bull. 73, 302-307 (2016)

24. Rao, B. M., Torabi, A. \& Varghese, O. K. Anodically grown functional oxide nanotubes and applications. MRS Commun. 375-396 (2016).

25. Varghese, O. K., Paulose, M. \& Grimes, C. A. Long vertically aligned titania nanotubes on transparent conducting oxide for highly efficient solar cells. Nat Nano 4, 592-597 (2009).

26. Lin, S., Li, D., Wu, J., Li, X. \& Akbar, S. A. A selective room temperature formaldehyde gas sensor using $\mathrm{TiO}_{2}$ nanotube arrays. Sensors Actuators B Chem. 156, 505-509 (2011).

27. $\mathrm{Lu}, \mathrm{H}$. F. et al. Amorphous $\mathrm{TiO}_{2}$ nanotube arrays for low-temperature oxygen sensors. Nanotechnology 19, 405504 (2008).

28. Perillo, P. M. \& Rodríguez, D. F. The gas sensing properties at room temperature of TiO2 nanotubes by anodization. Sensors Actuators B Chem. 171-172, 639-643 (2012).

29. Kwon, Y. et al. Enhanced ethanol sensing properties of $\mathrm{TiO}_{2}$ nanotube sensors. Sensors Actuators, B Chem. 173, 441-446 (2012).

30. Şennik, E., Çolak, Z., Kilinç, N. \& Öztürk, Z. Z. Synthesis of highly-ordered $\mathrm{TiO}_{2}$ nanotubes for a hydrogen sensor. Int. J. Hydrogen Energy 35, 4420-4427 (2010).

31. Varghese, O. K., Gong, D., Paulose, M., Ong, K. G. \& Grimes, C. A. Hydrogen sensing using titania nanotubes. Sensors Actuators B Chem. 93, 338-344 (2003).

32. Comini, E., Galstyan, V., Faglia, G., Bontempi, E. \& Sberveglieri, G. Highly conductive titanium oxide nanotubes chemical sensors. Microporous Mesoporous Mater. 208, 165-170 (2015).

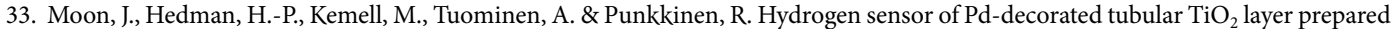
by anodization with patterned electrodes on $\mathrm{SiO}_{2} / \mathrm{Si}$ substrate. Sensors Actuators B Chem. 222, 190-197 (2016).

34. Persaud, K. In Electronic Noses and Tongues in Food Science (ed. Méndez, M. L. R.) 1-12 (Academic Press, 2016).

35. Goschnick, J. An electronic nose for intelligent consumer products based on a gas analytical gradient microarray. In Microelectronic Engineering 57-58, 693-704 (2001).

36. Richter, C. \& Schmuttenmaer, C. A. Exciton-like trap states limit electron mobility in $\mathrm{TiO}_{2}$ nanotubes. Nat Nano 5, 769-772 (2010).

37. Close, T., Tulsyan, G., Diaz, C. A., Weinstein, S. J. \& Richter, C. Reversible oxygen scavenging at room temperature using electrochemically reduced titanium oxide nanotubes. Nat Nano 10, 418-422 (2015).

38. Albu, S. P., Ghicov, A., Macak, J. M., Hahn, R. \& Schmuki, P. Self-organized, free-standing TiO ${ }_{2}$ nanotube membrane for flowthrough photocatalytic applications. Nano Lett. 7, 1286-1289 (2007).

39. Sysoev, V. V., Kiselev, I., Trouillet, V. \& Bruns, M. Enhancing the gas selectivity of single-crystal SnO $\mathrm{S}_{2}$ :Pt thin-film chemiresistor microarray by $\mathrm{SiO}_{2}$ membrane coating. Sensors Actuators B Chem. 185, 59-69 (2013).

40. Albu, S. P., Ghicov, A. \& Schmuki, P. Lift off strategies for self-organized $\mathrm{TiO}_{2}$ nanotube layers. ECS Trans 16, 195-202 (2008)

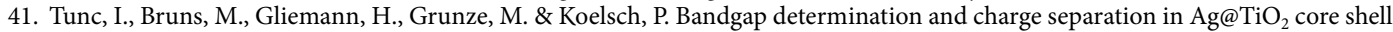
nanoparticle films. Surf. Interface Anal. 42, 835-841 (2010).

42. Albu, S. P. et al. Formation of double-walled $\mathrm{TiO}_{2}$ nanotubes and robust anatase membranes. Adv. Mater. 20, 4135-4139 (2008)

43. Lee, K., Mazare, A. \& Schmuki, P. One-dimensional titanium dioxide nanomaterials: nanotubes. Chem. Rev. 114, 9385-9454 (2014).

44. Nguyen, Q. A. S., Bhargava, Y. V., Radmilovic, V. R. \& Devine, T. M. Structural study of electrochemically synthesized TiO 2 nanotubes via cross-sectional and high-resolution TEM. Electrochim. Acta 54, 4340-4344 (2009).

45. Göpel, W., Rocker, G. \& Feierabend, R. Intrinsic defects of $\mathrm{TiO}_{2}(110)$ : interaction with chemisorbed $\mathrm{O}_{2}, \mathrm{H}_{2}, \mathrm{CO}_{\text {, and CO}}$. Phys. Rev. B 28, 3427-3438 (1983) 
46. Smith, K. E., Mackay, J. L. \& Henrich, V. E. Interaction of $\mathrm{SO}_{2}$ with nearly perfect and defect $\mathrm{TiO}_{2}(110)$ surfaces. Phys. Rev. B 35 , 5822-5829 (1987).

47. Zhu, K., Neale, N. R., Miedaner, A. \& Frank, A. J. Enhanced charge-collection efficiencies and light scattering in dye-sensitized solar cells using oriented $\mathrm{TiO}_{2}$ nanotubes arrays. Nano Lett. 7, 69-74 (2007).

48. Mardare, D. \& Rusu, G. I. Electrical conduction mechanism in polycrystalline titanium oxide thin films. J. Non. Cryst. Solids 356, 1395-1399 (2010).

49. Castro-Hurtado, I. et al. Formaldehyde sensing mechanism of $\mathrm{SnO}_{2}$ nanowires grown on-chip by sputtering techniques. RSC Adv. 6, 18558-18566 (2016)

50. Wolkenstein, T. Electronic Processes on Semiconductor Surfaces during Chemisorption (Consultants Bureau, 1991).

51. Hierlemann, A. \& Gutierrez-Osuna, R. Higher-order chemical sensing. Chem. Rev. 108, 563-613 (2008).

52. Sysoev, V. V., Kiselev, I., Frietsch, M. \& Goschnick, J. Temperature gradient effect on gas discrimination power of a metal-oxide thinfilm sensor microarray. Sensors 4, 37-46 (2004).

53. Korotcenkov, G. \& Cho, B. K. The role of grain size on the thermal instability of nanostructured metal oxides used in gas sensor applications and approaches for grain-size stabilization. Prog. Cryst. Growth Charact. Mater. 58, 167-208 (2012).

54. Barsan, N. \& Weimar, U. Conduction model of metal oxide gas sensors. J. Electroceramics 7, 143-167 (2001).

55. Kissine, V. V., Sysoev, V. V. \& Voroshilov, S. A. Individual and collective effects of oxygen and ethanol on the conductance of $\mathrm{SnO}_{2}$ thin films. Appl. Phys. Lett. 76, 2391-2393 (2000).

56. Tang, H., Prasad, K., Sanjinès, R., Schmid, P. E. \& Lévy, F. Electrical and optical properties of $\mathrm{TiO}_{2}$ anatase thin films. J. Appl. Phys. 75, 2042-2047 (1994)

57. Hattori, M. et al. Investigation of electrical transport in anodized single $\mathrm{TiO}_{2}$ nanotubes. Appl. Phys. Lett. 102, 1-5 (2013).

58. Diebold, U. The surface science of titanium dioxide. Surf. Sci. Rep. 48, 53-229 (2003).

59. Roberts, S. Dielectric constants and polarizabilities of ions in simple crystals and barium titanate. Phys. Rev. 76, 1215-1220 (1949).

60. Rothschild, A. \& Komem, Y. The effect of grain size on the sensitivity of nanocrystalline metal-oxide gas sensors. J. Appl. Phys. 95 (2004).

61. Cai, Y. \& Feng, Y. P. Review on charge transfer and chemical activity of $\mathrm{TiO}_{2}$ : mechanism and applications. Prog. Surf. Sci. 91, 183-202 (2016)

62. Parry, K. L. et al. ARXPS characterisation of plasma polymerised surface chemical gradients. Surf. Interface Anal. 38, 1497-1504 (2006).

63. Scofield, J. H. Hartree-Slater subshell photoionization cross-sections at 1254 and $1487 \mathrm{eV}$. J. Electron Spectros. Relat. Phenomena 8, 129-137 (1976).

64. Tanuma, S., Powell, C. J. \& Penn, D. R. Calculations of electron inelastic mean free paths. Surf. Interface Anal. 21, 165-176 (1994).

\section{Acknowledgements}

We thank V. Sakharov for technical assistance on material characterization and Prof. A. Kolmakov (NIST) for help in reviewing the manuscript. F.S.F., M.U.V., A.V. L., A.S.V., V.V.S. thank Russ. Ministry of Education and Science for a partial support by grants no. 16.1119.2017/4.6 and K3-2016-031 issued to MISiS. M.B. thanks German Fed. Ministry of Economics and Technology to support the K-Alpha + instrument. The authors thanks Karlsruhe Nano Micro Facility (KNMF, KIT, Germany), project 2016-016-012029.

\section{Author Contributions}

F.F., M.V. and V.S. conceived the idea and designed the experiments. M.V. contributed extensively to sample fabrication. A.L., A.V. contributed to gas sensing experiments. F.F., M.V., D.F., Ch.K., M.B. carried out the material characterization. F.F., V.S. contributed to the data analysis and discussion of mechanisms involved. All authors contributed to writing the paper.

\section{Additional Information \\ Supplementary information accompanies this paper at doi:10.1038/s41598-017-10495-8}

Competing Interests: The authors declare that they have no competing interests.

Publisher's note: Springer Nature remains neutral with regard to jurisdictional claims in published maps and institutional affiliations.

Open Access This article is licensed under a Creative Commons Attribution 4.0 International License, which permits use, sharing, adaptation, distribution and reproduction in any medium or format, as long as you give appropriate credit to the original author(s) and the source, provide a link to the Creative Commons license, and indicate if changes were made. The images or other third party material in this article are included in the article's Creative Commons license, unless indicated otherwise in a credit line to the material. If material is not included in the article's Creative Commons license and your intended use is not permitted by statutory regulation or exceeds the permitted use, you will need to obtain permission directly from the copyright holder. To view a copy of this license, visit http://creativecommons.org/licenses/by/4.0/.

(C) The Author(s) 2017 